\title{
Senologie, EHA \& ICML: Brustkrebs-Therapie und Hämatoonkologie im Fokus
}

Wenn es um die Behandlung von Brustkrebs geht, gilt die Jahrestagung der Deutschen Gesellschaft für Senologie als die bedeutendste interdisziplinäre Austausch- und Diskussionsplattform in Deutschland. Das ONKO-Internetportal sprach vor Ort mit Experten über die wichtigsten Themen des Kongresses.

$\mathrm{Zu}$ den Berichterstattungs-Schwerpunkten des ONKO-Internetportals zählen in diesem Jahr unter anderem die Verbesserung des Managements von Patienten mit Knochen- und ZNS-Metastasen sowie neue systemische Therapieansätze zur Behandlung bestimmter Subtypen des Mammakarzinoms, die Prof. Volkmar Müller (Hamburg) im Interview erläutert.

Der Stellenwert der Komplementärmedizin bei der Verbesserung der Lebensqualität onkologischer Patienten wird von Dr. Steffen Wagner (Saarbrücken) eingeordnet. Prof. Christian Jackisch (Offenbach) berichtet über die sich wandelnde Rolle der Chirurgie in der Brustkrebstherapie.

Alle Interviews zum Senologiekongress finden interessierte Ärzte unter www.krebsgesellschaft.de/senologiekonress2017 oder unter diesem QR-Code:

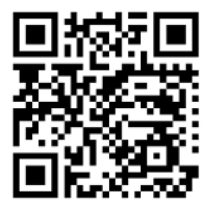

\section{Neue Daten vom EHA-Kongress und von der ICML}

Über die neusten Entwicklungen in der Diagnostik und Therapie des Multiplen Myeloms informiert das ONKO-Internetportal in einem State-of-the-Art-Gespräch vom EHA-Kongress 2017. Im Zentrum der Diskussion stehen insbesondere der Umgang mit neuen Wirkstoffen, die 24-Stunden-Urin-Messung, die Klassifizierung und Prognoseeinschätzung sowie die zunehmende Individualisierung der Therapie.

Dabei verweist Prof. Igor Blau (Berlin) auf die hierzulande einmalige Situation, dass alle zugelassenen Therapien auch für alle Patienten zur Verfügung stehen und von den Krankenkassen bezahlt werden. Prof. Harmut Goldschmidt (Heidelberg) mahnt an, über die medikamentöse Therapie nicht die persönliche Betreu- ung und das Gespräch mit dem Patienten zu vergessen. Prof. Monika Engelhardt (Freiburg) berichtet u. a. von neuen Scores zur eindeutigeren Klassifizierung der Erkrankung, um Über- und Untertherapien zu vermeiden.

Schwerpunktthemen bei den Video-Experteninterviews von der Jahrestagung der EHA sowie von der 14. International Conference on Malignant Lymphoma (ICML) sind in diesem Jahr neue Ansätze bei B-Zell-Lymphomen, bei Akuter und Chronischer Lymphatischer Leukämie und bei follikulären Lymphomen sowie der Bereich Stammzelltransplantation.

In Kongressberichten widmet sich das ONKO-Internetportal ausführlich einzelnen Studiendaten und -ergebnissen zu Leukämien und Lymphomen sowie zum Multiplen Myelom. Themen sind u. a. neue Kombinations-Therapien beim Multiplen Myelom, neue Daten zur Immunchemotherapie beim follikulären Lymphom und die Therapie mit Histonmethyltransferase-Inhibitoren bei Non-Hodgkin-Lymphomen.

Interessierte Ärzte können die komplette Kongressberichterstattung unter www.krebsgesellschaft.de/eha-icml2017 abrufen oder unter diesem QR-Code:

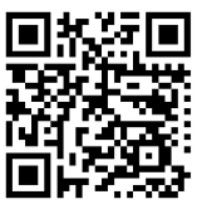

\section{Korrespondenzadresse}

\section{Dr. Pia Nitz}

Redaktion ONKO-Internetportal

In Kooperation mit der Deutschen Krebsgesellschaft e.V.

Tel. $030-810316112$

pia.nitz@dkg-web.de 